\title{
Flux to a Trap
}

Robert M. Ziff ${ }^{1}$

\begin{abstract}
The flux of particles to a single trap is investigated for two systems: (1) particles in 3D space which jump a fixed step length $l$ (the Rayleigh flight) and are adsorbed by a spherical surface, and (2) particles on a lattice, jumping to nearest neighbor sites, with a single adsorbing site. Initially, the particles are uniformly distributed outside the traps. When the jump length goes to zero, both processes go over to regular diffusion, and the first case yields the diffusive flux to a sphere as solved by Smoluchowski. For nonzero step length, the flux for large times is given by a modified form of Smoluchowski's result, with the effective radius replaced by $R-c l$, where $c=0.29795219$ and $c l$ is the Milne extrapolation length for this problem. For the second problem, a similar expression for the flux is found, with the effective trap radius a function of the lattice (sc, bcc, fcc) being considered.
\end{abstract}

KEY WORDS: Diffusion; trapping; random walks.

\section{INTRODUCTION}

The determination of the flux of particles diffusing toward an adsorbing surface or a trap lies at the foundation of the theory of aggregation and chemical kinetics. In the classic problem considered by Smoluchowski, ${ }^{(1)}$ the flux to a spherical, perfectly adsorbing surface is calculated by solving the diffusion equation, using the boundary condition $\rho \rightarrow 0$ and the initial condition that the particles are uniformly distributed outside the sphere with density $\rho_{0}$. The result is ${ }^{(1-3)}$

$$
\Phi(t)=4 \pi R D \rho_{0}\left(1+\frac{R}{(\pi D t)^{1 / 2}}\right)
$$

\footnotetext{
${ }^{1}$ Department of Chemical Engineering, University of Michigan, Ann Arbor, Michigan 48109-2136.
} 
where $D$ is the diffusion coefficient and $R$ is the radius of the sphere. This result, however, is not valid in two limits: for times of the order of, or less than, the fundamental time scale $\tau$ of the diffusing particle (the typical time for the particle to reverse its direction), and for $R$ of the order of, or smaller than, the corresponding diffusion length scale $l$. In these limits (1) is not valid because the diffusion equation is not applicable.

In this paper, we study corrections to (1) for two systems in which $l$ is of order $R$ : (1) a system of particles which jump a fixed step length $l$ but arbitrary direction in a three-dimensional (3D) continuum (the "Rayleigh flight"), and are adsorbed on a spherical trapping surface of radius $R$, and (2) particles on a lattice which hop to nearest-neighbor sites, adsorbed by a single trapping site. In both cases the particles jump in a discrete time interval $\tau$. These models were chosen because they correspond to the way diffusion is often carried out in computer simulations, and it is desirable to know what deviations from true Brownian motion result from their use.

The problem we consider is closely related to some classical problems in physics. Particles undergoing physical diffusion can be modeled as an Ornstein-Uhlenbeck (OU) process, described by the Fokker-Planck equation. ${ }^{(4)}$ The problem of finding the flux to an adsorbing boundary in this case is an old and well-studied one, ${ }^{(5-9)}$ and exact results have recently been found. ${ }^{(10,11)}$ A related problem is that of solving the neutron transport equation in the presence of an adsorbing boundary, first considered by Milne. ${ }^{(12-14)}$

For the continuum Rayleigh flight problem, we find that (1) is replaced by

$$
\Phi(t)=4 \pi R^{\prime} D \rho_{0}\left[1+\frac{R^{\prime}}{(\pi D t)^{1 / 2}}+\mathcal{O}\left(t^{-3 / 2}\right)\right]
$$

where $D=l^{2} / 6 \tau, R^{\prime} \equiv R-c l$, and $c \approx 0.29795219$, valid for $0<l \leqslant 2 R$. The $\mathcal{O}\left(t^{-3 / 2}\right)$ term also depends upon $l / R$, and disappears in the limit $l / R \rightarrow 0$. When $l$ is of the order of $R$, (2) predicts that there will be a significant change in the flux, both in the time-dependent part and the steady-state value. The distance $\beta=c l$ is the "Milne extrapolation length" ${ }^{(8,13,14)}$ and represents the distance inside the surface where the far steady-state solution $\rho(r)=\rho_{0}(r-R+\beta) / r$ extrapolates to $\rho=0$.

For particles hopping to a single adsorbing site on a $3 \mathrm{D}$ lattice, we show that (2) also obtains, but with $R^{\prime}$ proportional to the lattice spacing $l$. The value of the proportionality constant depends upon the lattice being considered. We consider the simple cubic (sc), body-centered cubic (bcc), and face-centered cubic (fcc) lattices. We also briefly consider 1D and 2D systems. 


\section{THE RAYLEIGH FLIGHT}

In this model, the particles jump a fixed distance $l$ in a discrete time step $\tau$, as illustrated in Fig. 1 . We will assume that $l \leqslant 2 R$. The probability density $\rho(\mathbf{r}, t)$ satisfies the discrete-time Markov equation

$$
\rho(\mathbf{r}, t+\tau)=\int W\left(\mathbf{r} \mid \mathbf{r}^{\prime}\right) \rho\left(\mathbf{r}^{\prime}, t\right) d \mathbf{r}^{\prime}
$$

where $W\left(\mathbf{r} \mid \mathbf{r}^{\prime}\right)$ is the jump probability from $\mathbf{r}^{\prime}$ to $\mathbf{r}$ in time $\tau$ and is given by $\delta\left(\left|\mathbf{r}-\mathbf{r}^{\prime}\right|-l\right) / 4 \pi l^{2}$. The initial condition is $\rho(\mathbf{r}, 0)=\rho_{0}$ for $r>R$, and $\rho(\mathbf{r}, 0)=0$ for $r \leqslant R$, and the boundary condition is $\rho(\mathbf{r}, t)=0$ for $r \leqslant R$ and all $t$, where $r=|\mathbf{r}|$.

Because of the spherical symmetry of the initial and boundary conditions, we need only be concerned with how the radial component of the position of a particle changes when it jumps. In the Appendix, we show that (3) implies that $f(r, t)=r \rho(\mathbf{r}, t)$ satisfies

$$
f(r, t+\tau)=\frac{1}{2 l} \int_{|r-l|}^{r+!} f\left(r^{\prime}, t\right) d r^{\prime}
$$

Thus $f$ is formally the solution to a one-dimensional problem in which a particle jumps uniformally within the interval $\pm l$ (when $r>l$ ). The function $f(r, t)$ satisfies the boundary condition $f(r, t)=0$ for $r \leqslant R$, and the initial condition

$$
f(r, 0)= \begin{cases}\rho_{0} r, & r>R \\ 0, & r \leqslant R\end{cases}
$$

To find the flux of particles into the sphere, we assume that a particle is adsorbed if its final radial distance after a jump falls within $R$, but that the particle remains free if the trajectory passes through the sphere and ends up outside of it, as illustrated in Fig. 1. (We call the latter grazing

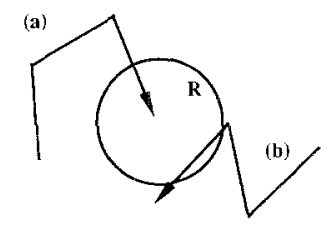

Fig. 1. The Rayleigh flight being intercepted by a spherical trap. In path (a), the particle is adsorbed because its final position is in the sphere. Path (b) shows a grazing trajectory, and (except in the discussion in Section 4) we assume that these particles are not adsorbed. 
trajectories.) In the Appendix, we show that the expression for the flux is then given by

$$
\Phi(t+\tau)=\frac{\pi}{l \tau} \int_{R}^{R+l}\left[R^{2}-\left(r^{\prime}-l\right)^{2}\right] f\left(r^{\prime}, t\right) d r^{\prime}
$$

When $f(r, t)$ changes slowly over distances of length $l$ and times of duration $\tau,(4)$ goes over to the diffusion equation with $D=l^{2} / 6 \tau$. We note that in the classical derivation of the diffusive flux to a sphere, it is also useful to introduce the same function $f(r, t)$, which formally reduces the problem to one-dimensional diffusion. ${ }^{(1)}$ When the diffusion equation is used, the flux at the spherical boundary is determined by $4 \pi R^{2} D(\partial \rho / \partial r)_{R}=$ $4 \pi D[r(\partial f / \partial r)-f]_{R}$.

It is convenient to introduce $x \equiv(r-R) / l, T \equiv t / \tau$, and $F(x, T) \equiv$ $f(R+l x, T \tau)$, where $T=1,2,3, \ldots$. Then (4)-(6) become

$$
\begin{aligned}
F(x, T+1) & =\frac{1}{2} \int_{x-1}^{x+1} F\left(x^{\prime}, T\right) d x^{\prime} \\
F(x, 0) & =\rho_{0}(l x+R) \\
\Phi[(T+1) \tau] & =\frac{4 \pi R D}{l}\left[a(T)-\frac{l}{R} b(T)\right]
\end{aligned}
$$

where

$$
\begin{aligned}
& a(T)=3 \int_{0}^{1}(1-x) F(x, T) d x \\
& b(T)=\frac{3}{2} \int_{0}^{1}(1-x)^{2} F(x, T) d x
\end{aligned}
$$

and $F(x, T)=0$ for $x<0$. In (9) we have introduced $D=l^{2} / 6 \tau$.

Next, we separate $F$ into two parts,

$$
F(x, t)=\rho_{0}\left[l F^{(1)}(x, t)+R F^{(2)}(x, t)\right]
$$

which satisfy the initial conditions

$$
\begin{array}{ll}
F^{(1)}(x, 0)=x & {[\text { i.c. }(1)]} \\
F^{(2)}(x, 0)=1 & \text { [i.c. }(2)]
\end{array}
$$

This decomposition of $F$ is useful because it allows us to consider (7) with these two dimensionless boundary conditions, rather than with (8). The flux is given by 


$$
\begin{aligned}
\Phi(T \tau) & =\rho_{0}\left[l \Phi^{(1)}(T)+R \Phi^{(2)}(T)\right] \\
& =4 \pi R D \rho_{0}\left[\phi^{(1)}(T)+\frac{R}{l} \phi^{(2)}(T)\right] \\
& =4 \pi R D \rho_{0}\left[a^{(1)}(T)-(l / R) b^{(1)}(T)+(R / l) a^{(2)}(T)-b^{(2)}(T)\right]
\end{aligned}
$$

where $\phi^{(i)}(T)=a^{(i)}(T)-(l / R) b^{(i)}(T)$, for $i=1,2$.

In summary, $F^{(1)}(x, T)$ and $F^{(2)}(x, T)$ follow iteratively for $T=1,2, \ldots$ from (7) and the initial conditions (12), and the flux follows from (13). The results for the first three interations of this procedure for i.c. (1) are

$$
\begin{aligned}
\phi^{(1)}(1) & =\frac{1}{2}\left(1-\frac{1}{4} \frac{l}{R}\right) \\
F^{(1)}(x, 1) & = \begin{cases}\left(1+2 x+x^{2}\right) / 4, & 0<x<1 \\
x, & 1<x\end{cases} \\
\phi^{(1)}(2) & =\frac{11}{16}\left(1-\frac{16}{55} \frac{l}{R}\right) \\
F^{(1)}(x, 2) & = \begin{cases}\left(7+12 x+6 x^{2}\right) / 24, & 0<x<1 \\
\left(8+12 x+6 x^{2}-x^{3}\right) / 24, & 1<x<2 \\
x, & 2<x\end{cases} \\
\phi^{(1)}(3) & =\frac{3}{4}\left(1-\frac{53}{180} \frac{l}{R}\right) \\
F^{(1)}(x, 3) & = \begin{cases}\left(60+100 x+42 x^{2}+4 x^{3}-x^{4}\right) / 192, & 0<x<1 \\
\left(73+92 x+48 x^{2}-8 x^{3}\right) / 192, & 1<x<2 \\
\left(81+84 x+54 x^{2}-12 x^{3}+x^{4}\right) / 192, & 2<x<3 \\
x, & 3<x\end{cases} \\
\phi^{(1)}(4) & =\frac{151}{192}\left(1-\frac{4 \cdot 391}{35 \cdot 151} \frac{l}{R}\right)
\end{aligned}
$$

and those for i.c. (2) are

$$
\begin{aligned}
\phi^{(2)}(1) & =\frac{3}{2}\left(1-\frac{1}{3} \frac{l}{R}\right) \\
F^{(2)}(x, 1) & = \begin{cases}(1+x) / 2, & 0<x<1 \\
1, & 1<x\end{cases} \\
\phi^{(2)}(2) & =\left(1-\frac{5}{16} \frac{l}{R}\right)
\end{aligned}
$$




$$
\begin{aligned}
& F^{(2)}(x, 2)= \begin{cases}(3+4 x) / 8, & 0<x<1 \\
\left(4+4 x-x^{2}\right) / 8, & 1<x<2 \\
1, & 2<x\end{cases} \\
& \phi^{(2)}(3)=\frac{13}{16}\left(1-\frac{4}{13} \frac{l}{R}\right) \\
& F^{(2)}(x, 3)= \begin{cases}\left(15+21 x+3 x^{2}-x^{3}\right) / 48, & 0<x<1 \\
\left(17+27 x-6 x^{2}\right) / 48, & 1<x<2 \\
\left(21+27 x-9 x^{2}+x^{3}\right) / 48, & 2<x<3 \\
1, & 3<x\end{cases} \\
& \phi^{(2)}(4)=\frac{7}{10}\left(1-\frac{5 \cdot 41}{7 \cdot 96} \frac{l}{R}\right)
\end{aligned}
$$

In the limit of $l / R$ small and $n$ large, we expect (13) to go over to (1). For that to occur, we must have $\phi^{(1)} \rightarrow 1$ and $\phi^{(2)} \sim(6 / \pi T)^{1 / 2}$. Clearly, many more time steps are needed to find how the $\phi^{(i)}$ deviate from this behavior for $l \geqslant R$. In the next section, we develop recurrence relations that allow the coefficients to the expression for the flux to be determined by a simple numerical procedure.

We note that the piecewise behavior of the $F^{(i)}(x, t)$ is reminiscent of Rayleigh's solution for the probability distribution of the position of a single particle following the same walk, as discussed by Chandrasekhar. ${ }^{(2)}$ In fact, that problem can also be treated by the procedure given here, changing only the initial and boundary conditions.

\section{NUMERICAL SOLUTION}

We define $F_{k}(x, t)(k=1,2,3, \ldots)$ as the functional form of $F$ in the interval $(k-1)<x<k$. Equation (7) implies that the $F_{k}$ satisfy

$$
\begin{aligned}
F_{k}(x, T+1)= & \frac{1}{2} \int_{x-1}^{k-1} F_{k-1}\left(x^{\prime}, T\right) d x^{\prime}+\frac{1}{2} \int_{k-1}^{k} F_{k}\left(x^{\prime}, T\right) d x^{\prime} \\
& +\frac{1}{2} \int_{k}^{x+1} F_{k+1}\left(x^{\prime}, T\right) d x^{\prime}
\end{aligned}
$$

where $F_{0}(x, t) \equiv 0$. Next, we expand each of the $F_{k}$ as a power series in $(k-x)$ :

$$
F_{k}(x, T)=\sum_{i=0}^{\infty} F_{k i}(T)(k-x)^{i}
$$


(This form leads to much simpler relations than a power series in $x$.) Inserting (17) into (16), we find the following simple recurrence relations for the $F_{k i}$ :

$$
\begin{aligned}
& F_{k i}(T+1)=\frac{1}{2 i}\left[F_{k-1, i-1}(T)-F_{k+1, i-1}(T)\right] \quad(i>0) \\
& F_{k 0}(T+1)=\sum_{i=0}^{\infty} \frac{1}{2(i+1)}\left[F_{k, i}(T)+F_{k+1, i}(T)\right]
\end{aligned}
$$

where $F_{0, i}(t)=0$. To calculate the flux, (9)-(10) is used with $F(x, T)$ replaced by $F_{1}(x, T)$, leading to

$$
\begin{aligned}
& a(T)=3 \sum_{i=0}^{\infty} \frac{F_{1 i}(T)}{(i+2)} \\
& b(T)=\frac{3}{2} \sum_{i=0}^{\infty} \frac{F_{1 i}(T)}{(i+3)}
\end{aligned}
$$

In terms of the $F_{k i}$, the two initial conditions (12) become

$$
\begin{aligned}
& F_{k i}^{(1)}(0)= \begin{cases}k, & i=0 \\
-1, & i=1 \\
0, & i>1\end{cases} \\
& F_{k i}^{(2)}(0)= \begin{cases}1, & i=0 \\
0, & i>0\end{cases}
\end{aligned}
$$

for all $k=1,2,3, \ldots$

Thus we have reduced the problem of finding the time-dependent density profile to solving the set of recurrence formulas (18) for the coefficients $F_{k i}^{(1)}(T)$ and $F_{k i}^{(2)}(T)$, starting with the initial values in (20). Once these coefficients are found, the flux is calculated from (13) and (19). This procedure is easily programmed on a computer, and in Table I we give the some of the resulting values of the coefficients $a(T)$ and $b(T) / a(T)$ for times up to $T=60$. Calculations were carried out in standard double-precision (16 significant digits). Analyzing these coefficients numerically, we find the following asymptotic expressions:

$$
\begin{aligned}
a^{(1)}(T) & =1-b^{(2)}(T) \\
b^{(1)}(T) / a^{(1)}(T) & =c-s c d T^{-3 / 2}+\mathcal{O}\left(T^{-2}\right) \\
a^{(2)}(T) & =s T^{-1 / 2}+(s / 20) T^{-3 / 2}+\mathcal{O}\left(T^{-5 / 2}\right) \\
b^{(2)}(T) / a^{(2)}(T) & =c+d T^{-1}+\mathcal{O}\left(T^{-2}\right)
\end{aligned}
$$


Table I. Values of the Coefficients in the Flux (32), for Time up to 60T

\begin{tabular}{rcccc}
\hline$T$ & $a^{(1)}(T)$ & $b^{(1)}(T) / a^{(1)}(T)$ & $a^{(2)}(T)$ & $b^{(2)}(T) / a^{(2)}(T)$ \\
\hline 1 & 0.500000 & 0.250000 & 1.50000 & 0.333333 \\
2 & 0.687500 & 0.290909 & 1.00000 & 0.312500 \\
3 & 0.750000 & 0.294444 & 0.812500 & 0.307692 \\
4 & 0.786458 & 0.295932 & 0.700000 & 0.305060 \\
5 & 0.810417 & 0.296582 & 0.624479 & 0.303586 \\
6 & 0.827800 & 0.296952 & 0.569048 & 0.302612 \\
7 & 0.841133 & 0.297181 & 0.526177 & 0.301926 \\
8 & 0.851784 & 0.297335 & 0.491733 & 0.301416 \\
9 & 0.860544 & 0.297444 & 0.463275 & 0.301023 \\
10 & 0.867914 & 0.297525 & 0.439248 & 0.300709 \\
20 & 0.907272 & 0.297812 & 0.309800 & 0.299317 \\
30 & 0.924467 & 0.297878 & 0.252737 & 0.298859 \\
40 & 0.934664 & 0.297905 & 0.218784 & 0.298631 \\
50 & 0.941603 & 0.297918 & 0.195636 & 0.298495 \\
60 & 0.946717 & 0.297927 & 0.178554 & 0.298404 \\
\hline
\end{tabular}

where $c=0.29795219, d=0.0270103$, and $s=(6 / \pi)^{1 / 2}$. To arrive at these results, various considerations were used. The identity in (21a) was observed to always hold, and although unproven, is undoubtedly true. The leading coefficient of $(21 \mathrm{c})$ is the value needed to recover (1) in the diffusive limit, and was verified numerically to six significant digits. The second term was found to be $s / 20$ to six significant figures, and we guess that it is exact. Both (21b) and (21d) were found to have the same leading behavior, the constant $c$. Note, however, that in $(21 \mathrm{~b})$, the first correction is of order $T^{-3 / 2}$, while in (21d), it is of order $T^{-1}$.

Putting the results (21) into (13), we find after some algebra

$$
\Phi(t)=4 \pi R D \rho_{0}\left[(R-c l) / R+(6 / \pi T)^{1 / 2}(R-c l)^{2} / R l+\mathcal{O}\left(T^{-3 / 2}\right)\right]
$$

and replacing $l^{2} / 6 \tau$ by $D$ and $R-c l$ by $R^{\prime}$, we are led to the result (2). The $\mathcal{O}\left(t^{-3 / 2}\right)$ term in (2), after more algebra, is found to be given by

$$
\frac{1}{20}\left(\frac{6}{\pi}\right)^{1 / 2} \frac{R^{\prime \prime}}{l}\left(\frac{\tau}{t}\right)^{3 / 2}=\frac{\pi}{120}\left(\frac{l}{R^{\prime \prime}}\right)^{2}\left(\frac{R^{\prime \prime}}{(\pi D t)^{1 / 2}}\right)^{3}
$$

where $R^{\prime \prime} \equiv R-(c+40 d) l \approx R-1.378 l$. This term goes to zero as $(l / R)^{2}$ for $l \rightarrow 0$ with $R, D$, and $t$ fixed. 


\section{ADDITIONAL COMMENTS ON THE RAYLEIGH PROBLEM}

There are many additional points that can be made about this model, which we briefly summarize here.

1. The steady-state distribution. In steady state the solution rapidly approaches $F(x, \infty)=x+c$ for large $x$. For $x$ near 0 , the density deviates from this straight line with oscillating behavior, as shown in Fig. 2. In contrast, the deviations for the OU particles are monotonic and negative. ${ }^{(8-10)}$ As $x \rightarrow 0$, the density approaches $F\left(0^{+}, \infty\right)=0.408245$ or $\rho(r)=$ $0.408245 \rho_{0} l / R$. [The time-dependent approach to the steady-state value is given by $F\left(0^{+}, T\right) \sim 0.408-0.17 T^{-1 / 2}$.] Thus, the density of particles at an adsorbing boundary does not go to zero, except in the limit of $l \rightarrow 0$.

The steady-state solution can be found efficiently by using $F(x, 0)=$ $x+c=F^{(1)}(x, 0)+c F^{(2)}(x, 0)$ as the initial condition in place of $(11)$; then, the final steady-state solution is approached very quickly. For example, $b(T) / a(T)=c+\mathcal{O}\left(T^{-5 / 2}\right)$. Using this result, we were able to determine the accurate value of $c$ given above, and to deduce that the coefficient of the first-order correction term of $(21 \mathrm{~b})$ is given by $-s c d$. Note that in steady state, (18a) becomes a recurrence relation for $F_{k i}(\infty)$.

The behavior of the steady state can also be investigated by noting that $F(x, \infty)=e^{-w x}$ is a solution to the integral equation (7) if $w$ satisfies

$$
\sinh w=w
$$

Besides the trivial solution $w=0$ corresponding to a constant $F$, there are solutions on the complex $w$ plane. Writing $w=u+i v$, we find $u$ and $v$

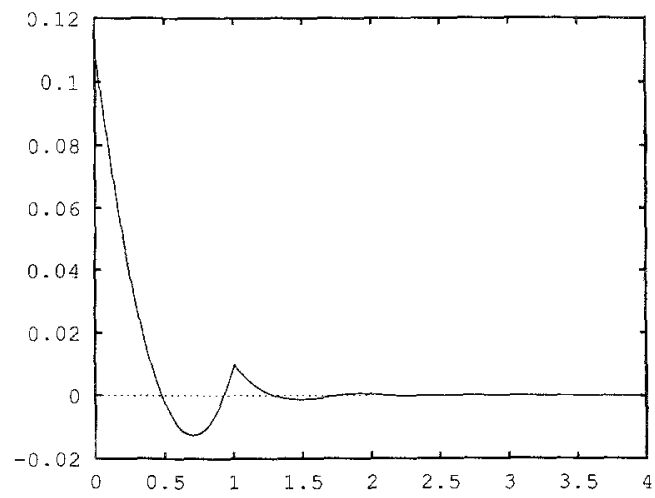

Fig. 2. The deviation in the steady-state density from the long-distance behavior $h(x)=$ $F(x)-x-c$, on a highly expanded scale. The actual density is found from $\rho(r)=\rho_{0}\{r-R+$ $c l+l h[(r-R) / l)]\} / r$. 
satisfy $u=\sinh u \cos v, v=\cosh u \sin v$, from which $v$ can be eliminated and then $u$ solved for. The first few solutions with the lowest values of $u$ are $w= \pm 2.76867828 \pm 7.49767628 i, \quad \pm 3.35220988 \pm 13.89995971 i$, $\pm 3.71676768 \pm 20.23851771 i$, and $\pm 3.98314164 \pm 26.55454727 i$. A linear combination of solutions with positive and negative $v$-both with positive $u$ so that the function decays in the positive $x$ direction-yields a solution with damped sinusoidal behavior $F(x, \infty)=e^{-u x} \cos (v x)$. However, these solutions only satisfy (7) for $x>1$, beyond the influence of the boundary condition. A linear combination is needed to solve (7) for $x<1$ also. We have not carried out such an analysis, but note that the dominant behavior is determined by the first root, which has an oscillation wavelength of $2 \pi / 7.49767628=0.83801768$ and rapid decay, consistent with the behavior seen in Fig. 2.

2. The diffusion-equation approach. A number of years ago, Collins and co-workers ${ }^{(15-18)}$ studied the flux using a hybrid approach in which the diffusion equation is assumed to be valid everywhere outside the trap, but the boundary condition is modified to

$$
\gamma\left(\frac{\partial \rho}{\partial r}\right)_{r=R}=\rho(R)
$$

to account for the kinetic boundary layer. The constant $\gamma$ must be determined by empirical arguments. The solution for an internal spherical boundary is given by ${ }^{(15)}$

$$
\begin{aligned}
\Phi & =4 \pi R^{\prime} D \rho_{0}\left[1+\left(R^{\prime} / \beta\right) \exp \left(D t / \beta^{2}\right) \operatorname{erfc}\left(D t / \beta^{2}\right)^{1 / 2}\right] \\
& \sim 4 \pi R^{\prime} D \rho_{0}\left[1+R^{\prime}(\pi D t)^{-1 / 2}-(\pi / 2) R^{\prime} \beta^{2}(\pi D t)^{-3 / 2} \cdots\right]
\end{aligned}
$$

as $t \rightarrow \infty$, where $\beta=\gamma /(1+\gamma / R)$ and here $R^{\prime}=R-\beta=R /(1+\gamma / R)$. Various prescriptions have been discussed to determine $\gamma^{(15.18)}$; however, to lowest order, they generally give the same result $\gamma=(l / 3)[1+\mathcal{O}(\varepsilon)]$, which implies $R^{\prime} / R=1-(1 / 3) \varepsilon+\mathcal{O}\left(\varepsilon^{2}\right)$, where $\varepsilon \equiv l / R$. When $\varepsilon$ is small, the difference between our expression for the flux and the one found above (asymptotically for large times) is just that our constant $c$ is replaced by $1 / 3$ here. In fact, the Collins-Kimball approach can be "fixed" to give the correct flux for Rayleigh-flight particles [up to $\mathcal{O}\left(t^{-3 / 2}\right)$ ] if $\gamma$ is taken to be $\mathrm{cl} /(1-c \varepsilon)$.

3. Continuous-time formulation. In a continuous-time formulation of this problem, the particles jump with an exponentially-distributed waiting time with mean $1 / \tau$. The jump length is still assumed to be fixed at $l$. The density $f_{c}(r, t)=r \rho_{c}(r, t)$ satisfies $^{(15)}$

$$
\frac{\partial f_{c}(r, t)}{\partial t}=-\frac{1}{\tau} f_{c}(r, t)+\frac{1}{2 \tau l} \int_{|r-l|}^{r+l} f_{c}\left(r^{\prime}, t\right) d r^{\prime}
$$


The solution of (27) is related to the discrete-time density $f(r, t)$ by

$$
f_{c}(r, t)=e^{-t / \tau} \sum_{m=0}^{\infty} \frac{(t / \tau)^{m}}{m !} f(r, m \tau)
$$

since, when (28) is inserted into (27), $f(r, t)$ is found to satisfy (4). The fluxes are related to each other in a similar way as in (28). It can be shown that if the discrete-time flux is given by (2), then the continuous-time flux will be identical in the zeroth and first orders, and only the $\mathcal{O}\left(t^{-3 / 2}\right)$ (and higher-order) terms will differ. Thus, (2) is valid in a continuous-time Rayleigh walk system as well.

4. Grazing trajectories. So far, we have ignored grazing trajectories. In the Appendix, we show that such trajectories contribute the following term, in addition to (6), to the flux:

$$
\Phi_{g}(t+\tau)=\frac{\pi}{l \tau} \int_{R}^{\left(R^{2}+l^{2}\right)^{1 / 2}}\left[l-\left(r^{\prime 2}-R^{2}\right)^{1 / 2}\right]^{2} f\left(r^{\prime}, t\right) d r^{\prime}
$$

While including adsorption of grazing trajectories will make the time evolution much more complicated, since these particles must be subtracted from the density distribution, we can get an idea of their importance by considering the first time-step only, where $f(r, 0)=\rho_{0} r$. Then, (29) gives

$$
\Phi_{g}(\tau)=\pi R^{2} \rho_{0}(l / \tau)\left(\varepsilon^{2} / 12\right)
$$

In contrast, (6) gives [see (13), (14a), and (15a)]

$$
\Phi(\tau)=\pi R^{2} \rho_{0}(l / \tau)\left(1-\varepsilon^{2} / 12\right)
$$

Thus, the effect of the grazing term is of second order in $\epsilon$, compared to the leading term in $\Phi(\tau)$.

It is interesting that (30) exactly cancels out the second-order term in (31), so that their sum is simply $\Phi(\tau)=\pi R^{2} \rho_{0}(l / \tau)$. The same expression follows from a simple kinetic theory calculation, since $4 \pi R^{2}$ is the surface area and $l / 2 \tau$ is the average normal component of the velocity of the particles moving toward the surface, which comprise half of the particles in the system. This argument, however, assumes that the surface is flat. The above calculation shows that this expression remains valid for a spherical surface as well, when the grazing trajectories are included in the flux.

\section{FLUX FOR DIFFUSION ON A LATTICE}

Here we briefly consider the problem of the flux where the diffusing particles are jumping from site to neighboring site on a lattice at each unit 
time step. The trap is a single adsorbing site, and the initial condition is a uniform distribution of particles outside the trap with concentration unity. The flux $\phi_{T}$ is equivalent to the number of possible paths from all sites to the trap, such that the paths arrive at the trap for the first time at time $T$, multiplied by the weight of each path $(1 / Z)^{T}$, where $Z$ is the coordination number of the lattice. Reversing the time, it can be seen that the number of these paths is the same as the number of paths that leave the trap and never return during $T$ time steps. Thus, we can write $\phi_{T}=1-\sum_{j=1}^{T} f_{j}$, where $f_{j}$ is the probability of first return in $j$ time steps. The generating function of $\phi_{T}$ is then

$$
\begin{aligned}
\Phi(z) & \equiv \sum_{T=1}^{\infty} z^{T} \phi_{T} \\
& =\frac{1-F(z)}{1-z}=\frac{1}{(1-z) P(z)}
\end{aligned}
$$

where $F(z) \equiv \sum_{j=1}^{\infty} z_{j}^{j} f_{j}$. In the last expression, we used $F(z)=1-1 / P(z)$, where $P(z) \equiv \sum_{j=1}^{\infty} z^{j} p_{j}$ and $p_{j}$ is the probability of return to the origin at $j$ time steps, irrespective of whether it had been there before. ${ }^{(19)}$ Note that (32) is quite similar to the expression for the generating function $S(z)$ of the number of distinct sites $s_{T}$ visited at time step $T: S(z)=$ $1 /\left[(1-z)^{2} P(z)\right] .{ }^{(20)}$ This relation implies $\phi_{T}=s_{T}-s_{T-1}$. Note also that $f_{T}=-\phi_{T}+\phi_{T-1}$.

For a 1D lattice, we have $p_{2 n}=2^{-2 n}\left(\begin{array}{c}2 n \\ n\end{array}\right)$, implying $P(z)=\left(1-z^{2}\right)^{-1 / 2}$ and thus $\Phi(z)=(1+z)\left(1-z^{2}\right)^{-1 / 2}$, which implies that $\phi_{2 n}=\phi_{2 n+1}=p_{2 n}$. The latter result implies the asymptotic behavior $\phi_{T} \sim 2(D / \pi T)^{1 / 2}$, where $D=1 / 2$ is the diffusion coefficient in $1 \mathrm{D}$ for a system with $l=1$ and $\tau=1$. Indeed, this flux is identical to the (complete) expression for the flux calculated from diffusion equation in a $1 D$ system in which particles are being adsorbed on both sides of the trap (the factor of 2 in $\phi_{T}$ above).

For a 2D square lattice, $p_{2 n}=4^{-2 n}\left(\begin{array}{c}2 n \\ n\end{array}\right)^{2}$ and $P(z) \sim-(1 / \pi) \ln (1-z)$, implying $\phi_{T} \sim \pi / \ln T$. Higher-order terms can be deduced using the methods of ref. 21 , yielding

$$
\phi_{T} \sim \pi\left(\frac{1}{\ln (8 T)+\gamma}-\frac{\pi^{2} / 6}{[\ln (8 T)+\gamma]^{3}}-\frac{2 \zeta(3)}{[\ln (8 T)+\gamma]^{4}} \ldots\right)
$$

where (here) $\gamma=0.55721 \ldots$ is Euler's constant. There are correction terms of order $1 /(T \ln T)$ to (33). Comparison to the solution to the $2 \mathrm{D}$ diffusion equation with an adsorbing disk, ${ }^{(22)}$

$$
\phi_{T} \sim 4 \pi \rho_{0} D\left[\frac{1}{\ln \left(4 D t / R^{2}\right)-\gamma}+\mathcal{O}\left(\frac{1}{\ln \left(4 D t / R^{2}\right)-\gamma}\right)^{3}\right]
$$


yields $D=1 / 4$, as expected for a $2 \mathrm{D}$ system with $l=1$ and $\tau=1$, and $R=e^{-\gamma} 8^{-1 / 2} \approx 0.1985$. The latter gives the effective size of the sphere of influence of the trap, in units of the lattice spacing. If one thinks of the problem as one in which all particles and the trap are spheres of the same size, then the radius of those spheres will be $R / 2$, a number much smaller than the lattice spacing.

In 3D, the asymptotic behavior of $P(z)$ is well known for the sc, fcc, and bec lattices. ${ }^{(20)}$ For the sc lattice, for example, we find

$$
\Phi(z) \sim \frac{1}{P(1)(1-z)}+\frac{3^{3 / 2}}{2^{1 / 2} \pi P(1)^{2}(1-z)^{1 / 2}}+\cdots
$$

as $z \rightarrow 1$, where $P(1)=1.5163860592$, implying

$$
\phi_{T} \sim \frac{1}{P(1)}+\left(\frac{27}{2 \pi^{3} T}\right)^{1 / 2} \frac{1}{P(1)^{2}}+\mathcal{O}\left(T^{-3 / 2}\right)
$$

This result can be written in the form of (2), yielding $R^{\prime}=1 /[4 \pi D P(1)]=$ 0.31487 and $D=1 / 6$, which is in accord with the formula $D=l^{2} / 6 \tau$. This value of $R^{\prime}$ gives the effective size of the trap, in units of the lattice spacing.

A similar calculation for the bcc lattice yields $D=1 / 2$ and $R^{\prime}=1 /[4 \pi D P(1)]=0.11424$, using the known value of $P(1)$. This value of $D$ is in accord with the formula $l^{2} / 6 \tau$ because here the nearest neighbors are diagonal segments of length $l=\sqrt{3}$ from the corners to the center of the unit cube (whose side is of length 2). The effective size of the particle $R^{\prime}$ is 0.11424 of the lattice spacing (unity), or 0.06596 of the jump length $l$. For the fcc lattice, where nearest neighbors are along diagonals on the face of the cube and are a distance $l=\sqrt{2}$ apart, we have $D=1 / 3$ and $R^{\prime}=$ $1 /[4 \pi D P(1)]=0.31487=0.22265$ l. That $(2)$ holds for all these lattices implies that there is a general connection between the first-order term in the expansion of $P(z)$ for $z \rightarrow 1$, and the nearest-neighbor spacing.

Thus, for diffusion on a lattice, a trap is effectively a sphere with a radius that is much smaller than the lattice spacing. A consequence of this is that the trapping probability for particles on a lattice is much less than it would be in a continuum, for particles whose size is the same as the spacing of the lattice. On a lattice, a particle near a trap has a much higher probability of escaping-by making a big jump away from the trap than if the particle were in a continuum.

Finally, we discuss briefly a generalization to the case where the particles are undergoing persistent motion. We consider a 1D lattice of unit spacing with a trap at the origin, in which the diffusing particles continue in the same direction with probability $1-y$, and reverse direction with 
probability $y$-so that the velocity, rather than the position, is a Markov process. When $y=1 / 2$, the standard random walk considered above is recovered. The mean square displacement of a particle in $T$ time steps is given by ${ }^{(23)}$

$$
\left\langle(\Delta x)^{2}\right\rangle=T+\frac{1-2 y}{2 y^{2}}\left[2 y T-1+(1-2 y)^{T}\right]
$$

which implies $D=(1-y) / 2 y$. The flux at time $2 n$ is given by $\phi_{2 n}=$ $(1-y) p_{2 n} / y$, where $p_{2 n}$ is the return probability, and for large $T$,

$$
\phi_{T} \sim 2\left(\frac{2(1-y)}{\pi y T}\right)^{1 / 2}\left[1-\frac{1}{T}\left(\frac{1}{2 y(1-y)}-1\right)+\cdots\right]
$$

(The correction term above is valid for even $T$ only.) The leading term agrees with the flux calculated from the $1 \mathrm{D}$ diffusion equation, $\phi_{T}=$ $2(D / \pi T)^{1 / 2}$. When $y \rightarrow 0$ and $T \rightarrow \infty$ such that $y T$ is a constant, the particles move at constant speed and reverse directions after traversing an exponentially distributed distance.

\section{CONCLUSIONS}

Thus, we have found that $(2)$ is a general form for the flux to a trap in $3 \mathrm{D}$ for two different kinds of random walk processes. In (2), the same constant $R^{\prime}$ appears in both the steady-state and first-order terms. The generality of (2) can be understood by observing that the diffusion equation will be essentially valid far from a trap where the density of particles varies slowly with time and position. The net effect of the trap is therefore reduced to knowing its effective radius $R^{\prime}$ or the Milne extrapolation length. For the case of the Rayleigh flight, we have found this length numerically. Perhaps an analytic solution for this problem can be foundas has been done for the case of OU particles. ${ }^{(8,9)}$

When diffusion is simulated by a Rayleigh flight, it follows from (2) that significant errors will be introduced in the trapping probability unless $l / R \ll 1$. Practically, however, making $l$ so small generally slows down a simulation severely. A practical solution to this problem is to make the jump length depend upon the distance to the nearest trap. ${ }^{(24,25)}$ On the lattice, the error in the trapping probability can be corrected to some degree by making the probability of jumping to a site dependent upon whether that site is bordering a trap.

For future work, it would be interesting to study the behavior when the jump length is not constant, especially when the variance is infinite (the Lévy flight). 


\section{APPENDIX}

We define $P(r, t) d r \equiv 4 \pi r^{2} \rho(r, t) d r$ as the probability that a particle falls within the radial shell $(r, r+d r)$ about the origin. $P$ satisfies

$$
P(r, t+\tau)=\int w\left(r \mid r^{\prime}\right) P\left(r^{\prime}, t\right) d r^{\prime}
$$

where $w\left(r \mid r^{\prime}\right) d r=$ the probability of a particle jumping from radial distance $r^{\prime}$ to the interval $(r, r+d r)$ in one step. First consider the case that $l \leqslant r^{\prime}$. From Fig. 3, one can see that $w$ is given by

$$
\begin{aligned}
w\left(r \mid r^{\prime}\right) d r & =\frac{\text { surface area in region }(r, r+d r)}{\text { total surface area of sphere }} \\
& =\frac{2 \pi l \sin \theta l d \theta}{4 \pi l^{2}}=\frac{1}{2} \sin \theta d \theta
\end{aligned}
$$

Now, by the law of cosines, $r^{2}=\left(r^{\prime}\right)^{2}+l^{2}-2 r^{\prime} l \cos \theta$, and for fixed $r^{\prime}$, $2 r d r=2 r^{\prime} l \sin \theta d \theta$ or $\sin \theta d \theta=r d r /\left(r^{\prime} l\right)$. Thus,

$$
w\left(r \mid r^{\prime}\right) d r=\frac{r d r}{2 r^{\prime} l}, \quad\left|r^{\prime}-l\right|<r<\left(r^{\prime}+l\right)
$$

as given by Collins and Kimball. ${ }^{(15)}$ The absolute value bars above make this expression valid for $l>r^{\prime}$ also. Then, inserting (A3) into (A1) and introducing $f(r, t)=P(r, t) / 4 \pi r$, one finds the kinetic equation (7).

We note that $w$ is properly normalized:

$$
\int_{\left|r^{\prime}-l\right|}^{r^{\prime}+l} w\left(r \mid r^{\prime}\right) d r=\frac{1}{2 r^{\prime} l} \int_{\left|r^{\prime}-l\right|}^{r^{\prime}+l} r d r=1
$$

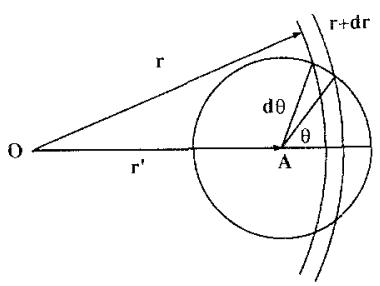

Fig. 3. A particle jumping from point $A$, at a distance $r^{\prime}$ from the origin, lands anywhere on the surface of the sphere of radius $l$ with equal probability. Equation (A2) gives the probability that the particle is on that part of the spherical surface whose radial distance from the origin falls in the interval $(r, r+d r)$. 
The flux of particles whose final radial distance falls within $R$ is given by

$$
\begin{aligned}
\Phi(t+\tau) & =\frac{1}{\tau} \int_{R}^{\infty} d r^{\prime} \int_{0}^{R} d r w\left(r \mid r^{\prime}\right) P\left(r^{\prime}, t\right) \\
& =\frac{2 \pi}{l \tau} \int_{R}^{R+l} d r^{\prime} f\left(r^{\prime}, t\right) \int_{\left|r^{\prime}-l\right|}^{R} d r r \\
& =\frac{\pi}{l \tau} \int_{R}^{R+l}\left[R^{2}-\left(r^{\prime}-l\right)^{2}\right] f\left(r^{\prime}, t\right) d r^{\prime}
\end{aligned}
$$

where we have assumed $l \leqslant 2 R$. (When $l>2 R$, the bracketed term in the last integral above must be replaced by zero whenever its value becomes negative.) For the adsorption of grazing trajectories, the flux contains the following additional term:

$$
\begin{aligned}
\Phi_{g}(t+\tau) & =\frac{1}{\tau} \int_{R}^{\left(R^{2}+l^{2}\right)^{1 / 2}} d r^{\prime} \int_{R}^{r_{0}} d r w\left(r \mid r^{\prime}\right) P\left(r^{\prime}, t\right) \\
& =\frac{2 \pi}{l \tau} \int_{R}^{\left(R^{2}+l^{2}\right)^{1 / 2}} d r^{\prime} f\left(r^{\prime}, t\right) \int_{R}^{r_{0}} d r r \\
& =\frac{\pi}{l \tau} \int_{R}^{\left(R^{2}+l^{2}\right)^{1 / 2}}\left[l-\left(r^{\prime 2}-R^{2}\right)^{1 / 2}\right]^{2} f\left(r^{\prime}, t\right) d r^{\prime}
\end{aligned}
$$

where the maximum value of $r^{\prime}$ for a grazing trajectory to occur is $\left(R^{2}+l^{2}\right)^{1 / 2}$, and $r_{0}$ defined by $r_{0}^{2}=R^{2}+\left[l-\left(r^{\prime 2}-R^{2}\right)^{1 / 2}\right]^{2}$ is the maximum radial distance of the final position of a grazing trajectory for a particle jumping from radial distance $r^{\prime}$ from the origin.

\section{ACKNOWLEDGMENTS}

The author thanks Charles Doering, George Weiss, and M. M. R. Williams for useful comments, and acknowledges National Science Foundation grant DMR-861973.

\section{REFERENCES}

1. M. Smoluchowski, Phys. Z. 17:557, 585 (1916).

2. S. Chandrasekhar, Rev. Mod. Phys. 15:1 (1943) [reprinted in Noise and Stochastic Processes, N. Wax, ed. (Dover, 1954)].

3. G. H. Weiss, J. Stat. Phys. 42:3 (1986).

4. G. E. Uhlenbeck and L. S. Ornstein, Phys. Rev. 36:823 (1930).

5. M. C. Wang and G. E. Uhlenbeck, Rev. Mod. Phys. 17:323 (1940). 
6. S. Harris, J. Chem. Phys. 75:3103 (1981); 77:934 (1982).

7. K. Razi Naqvi, K. J. Mork, and S. Waldenstrom, Phys. Rev. Lett. 49:304 (1982).

8. M. A. Burschka and U. M. Titulaer, J. Stat. Phys. $25: 569$ (1981).

9. U. M. Titulaer, J. Stat. Phys. 37:589 (1984).

10. T. W. Marshall and E. J. Watson, J. Phys. A 20:1345 (1987); 21:4241 (1988).

11. P. S. Hagan, C. R. Doering, and C. D. Levermore, SIAM J. Appl. Math. 49:1480 (1989).

12. E. W. Larsen and J. B. Keller, J. Stat. Phys. $15: 75$ (1974).

13. K. M. Case and P. W. Zwiefel, Linear Transport Theory (Addison-Wesley, Reading, Massachusetts, 1967).

14. M. M. R. Williams, The Slowing Down and Thermalization of Neutrons (North-Holland, Amsterdam, 1966).

15. F. C. Collins and G. E. Kimball, Ind. Eng. Chem. 41:2551 (1949).

16. F. C. Collins and G. E. Kimball, J. Colloid Sci. 4:425 (1949).

17. F. C. Collins, J. Colloid Sci. 5:499 (1950).

18. H. L. Frisch and F. C. Collins, J. Chem. Phys. 20:1797 (1952); 21:2158 (1953).

19. W. Feller, An Introduction to Probability Theory and Its Applications (Wiley, New York, 1951).

20. E. W. Montroll and G. H. Weiss, J. Math. Phys. 6:167 (1965).

21. F. S. Henyey and V. Seshadri, J. Chem. Phys. 76:5530 (1982).

22. H. S. Carslaw and J. C. Jaeger, Conduction of Heat in Solids, 2nd ed. (Oxford University Press, Oxford, 1959).

23. R. M. Ziff, X. P. Kong, and E. G. D. Cohen, Phys. Rev. A 44:2410 (1991).

24. P. Meakin, J. Phys. A 18:L661 (1985).

25. C. A. Miller, I. C. Kim, and S. Torquato, J. Chem. Phys. 94:5592 (1991). 